\title{
REDES DE MUJERES SIN VIOLENCIA: EMPODERAMIENTO Y TRANSFORMACIÓN SOCIAL DESDE LOS DERECHOS HUMANOS DE LAS MUJERES
}

Networks of Women Without Violence: Empowerment and Social Transformation from the Human Rights of Women

\author{
Mónica Maciel Méndez Morales \\ Directora del Instituto Municipal de las Mujeres de León, \\ Guanajuato, México \\ monica.immujeresleon@gmail.com
}

\section{Cinthia Aida Valenzuela Balderas \\ Profesionista de Redes de Mujeres cinthiaaida@gmail.com}

\begin{abstract}
RESUMEN: A partir de la experiencia del programa de Redes de Mujeres sin Violencia implementado por el Instituto Municipal de las Mujeres en el municipio de León, Guanajuato, México y la metodología utilizada, este artículo plantea dar a conocer uno de los principales derechos humanos de las mujeres: el derecho a una vida libre de violencia, como un pilar fundamental en la creación de acciones y políticas públicas con perspectiva de género. Se establece cómo surge el programa de Redes de Mujeres sin Violencia desde el año 2011 en el Municipio de León, el cual se ha constituido como una experiencia de educación popular dirigida a mujeres de colonias y comunidades rurales, la cual ha contribuido al empoderamiento y la transformación social de éstas y sus entornos por medio de procesos de formación y capacitación en los cuales se comparte información relacionada con la igualdad, los derechos humanos, la prevención y el acompañamiento de la violencia contra las mujeres. Además, se recuperan algunas vivencias y experiencias de las mujeres a fin de contextualizar la importancia de este programa en sus vidas cotidianas.
\end{abstract}


Palabras clave: Redes de mujeres, violencia de género, violencia contra las mujeres, participación ciudadana, sororidad.

RESUM: A partir de l'experiència del programa de Xarxes de Dones sense violència implementat per l'Institut Municipal de les Dones en el municipi de Lleó, Guanajuato, Mèxic i la metodologia utilitzada, aquest article planteja donar a conéixer un dels principals drets humans de les dones: el dret a una vida lliure de violència, com un pilar fonamental en la creació d'accions i polítiques públiques amb perspectiva de gènere. S'estableix, com sorgeix el programa de Xarxes de Dones sense violència des de l'any 2011 en el Municipi de Lleó el qual s'ha constituït com una experiència d'educació popular dirigida a dones de colònies i comunitats rurals la qual ha contribuït a l'apoderament $\mathrm{i}$ la transformació social d'aquestes i els seus entorns per mitjà de processos de formació i capacitació en els quals es comparteix informació relacionada amb la igualtat, els drets humans, la prevenció i l'acompanyament de la violència contra les dones. A més, es recuperen algunes vivències i experiències de les dones a fi de contextualitzar la importància d'aquest programa en les seues vides quotidianes.

Paraules clau: Xarxes de dones, violència de gènere, violència contra les dones, participació ciutadana, sororitat.

ABSTRACT: Based on the experience and methodology of the Network of Women Free From Violence program implemented by the Institute for Women of the City Council of León, Guanajuato, Mexico, this article aims to raise awareness about one of the main human rights of women - the right to a life free of violence - as a fundamental pillar in creating public actions and policies with a gender perspective. The paper describes how the Network of Women Free From Violence program was set up in 2011 in the Municipality of León, and how it has since become established as a grassroots education experience aimed at women in the neighborhoods and rural communities. The program has contributed to the empowerment and social transformation of these women and their environments through training and skill-building processes in which information is shared on equality, human rights, prevention of violence against women, and support for victims. Some of the women's 
experiences are also related to contextualize the importance of this program in their daily lives.

KEYWORDs: Women's networks, gender violence, violence against women, citizen participation, sisterhood.

\section{Introducción}

María $^{1}$ no sabía qué hacer. Sandra, su hija de 16 años, llevaba meses acosada por su ex pareja. Un joven adicto a las drogas mucho mayor que ella. El ex novio estaba aferrado a Sandra como si fuera parte de su propiedad. María, habitante de una colonia popular de León, Guanajuato, estaba consciente del peligro que significaba que su hija estuviera cerca de un consumidor habitual de drogas en el violento entorno de la ciudad. Un día, María regresó a su casa después de sus labores cotidianas y encontró el cuerpo de su hija. Sandra había sido violada y asesinada. En el lugar de los hechos se encontró una prenda propiedad del ex novio. La investigación criminal se inició de inmediato. Entre el dolor por el suceso, la confusión sobre la forma en la que el procedimiento legal se desarrolla y la soledad, María siente que se encuentra en un callejón sin salida. Para navegar por el sistema de justicia penal, María requiere necesariamente de asistencia. Su deseo de justicia no siempre es entendido de la misma forma por las autoridades. María requirió de un acompañamiento especializado durante todo el caso que le permitiera conocer con toda claridad sus derechos. María demanda justicia, no ruega por ella. El juicio en contra del ex novio concluyó tres años después con una sentencia condenatoria y ahora se van a resolver las apelaciones. Pero la relación con las autoridades solamente es uno de los obstáculos de María. María enfrenta sola la tragedia. Su entorno y su comunidad tampoco parecen tener claro qué hacer o buscan cómo salir del paso. Desde luego tampoco faltan quienes aprovechan la ocasión para re victimizarlas a ella y a su hija.

1. En todos los casos presentados a lo largo del texto, se utilizan nombres ficticios. 
Al final de cuentas es culpa de ellas por meterse con quien no debían. Todos lo vimos, era tan fácil dejarlo. Pero no lo hicieron a tiempo. Poco a poco, la narrativa de la culpa se impone sobre ellas: madre e hija. La narrativa que sustenta a la normalización de la violencia contra las mujeres se termina por imponer. Ahora solo queda la madre para enfrentarlo. Provista de su decisión de llevar las cosas hasta el final, pero sin herramientas, sin apoyos en la comunidad y sin solidaridad. Sola.

El caso de María no es único. En la Ciudad de León Guanajuato, al igual que en muchas otras partes, ocurren homicidios, feminicidios y otras formas de violencia en contra de las mujeres. Expresiones de violencia que pueden pasar desapercibidas o que son tratadas como otro asunto más. Heridas que nunca van a cicatrizar. Heridas que van a mitigarse en soledad. El programa Redes de Mujeres se enfoca precisamente en tratar de ayudar a prevenir estos problemas. Es una iniciativa que busca generar lazos de sororidad, comunidad y participación entre las mujeres. Los objetivos son muchos, pero uno de los más claros tiene que ver con la necesidad de visibilizar la violencia en contra de las mujeres, entenderla como un fenómeno que afecta a todas las personas, y dar herramientas que contribuyan al empoderamiento de las víctimas para poder enfrentarla y prevenirla. Este trabajo pretende exponer el contexto, las líneas esenciales de operación del programa y sus resultados.

En las líneas siguientes vamos a empezar por señalar los problemas más frecuentes de violencia que enfrentan las mujeres en León, Guanajuato. Para tal efecto, empleamos el marco jurídico de los derechos humanos de las mujeres. A partir de ahí tomamos el concepto de violencia en contra de las mujeres y lo describimos desde el punto de vista teórico, así como su incidencia en la ciudad de León. Esto nos permite caracterizar los problemas que enfrentamos e introducir la concepción del derecho a una vida libre de violencia como el eje central de una política pública con perspectiva de género y focalizada en prevenir y atender las agresiones que cotidianamente enfrentan las mujeres.

En la tercera parte, nos ocupamos del programa Redes de Mujeres sin Violencia. El programa se presenta como una acción del Instituto Municipal de las Mujeres de León, Guanajuato, diseñada para visibilizar, prevenir y atender los casos de violencia en contra de las mujeres. La novedad aquí consiste en 
involucrar directamente a las mujeres en el diagnóstico de los problemas que enfrentan en las colonias y comunidades, en generar propuestas de acción $\mathrm{y}$, sobre todo, en construir sororidad entre ellas, damos cuenta de las evaluaciones que se han hecho al programa Redes de Mujeres, sus logros y el reconocimiento internacional.

Finalmente, presentamos algunas conclusiones sobre la utilidad de esta experiencia como una herramienta para mejorar la vida de las mujeres.

\section{Marco Jurídico}

Los derechos humanos constituyen un pilar fundamental para el ejercicio de la ciudadanía de las mujeres, pues implica que se les reconozca como sujetas de derechos para que estos se promuevan, respeten, protejan y garanticen, desde la perspectiva general de los derechos humanos. Por lo tanto, al traducirse en la legislación y hacerse efectivos, las mujeres pueden exigirlos.

¿Por qué hablar de Derechos Humanos de las Mujeres en específico? La respuesta es muy clara. En nuestro contexto, las afectaciones a las mujeres permanecen generalmente invisibilizadas. La discriminación, la violencia y la desigualdad son mucho más frecuentes y evidentes de lo que estamos dispuestas a aceptar. Sin embargo, ahí están. Con ese manto de invisibilidad que les da la cotidianeidad. Por ello, es necesario empezar con un discurso que empodere a las mujeres desde lo más básico: sus derechos humanos.

Cuando Olympe de Gouges redactó de la Declaración de los Derechos de la Mujer y de la Ciudadanía en 1971, se enfocó en la necesidad de construir libertad e igualdad de las mujeres frente a ellas mismas y a los hombres. A partir de entonces, fue necesario empezar a revisar el contenido y los alcances de los llamados derechos del hombre. El cambió exigió pasar del «Todos los hombres nacen libres e iguales en dignidad y derechos [...]», a mujeres y hombres tienen los mismos derechos. Sin embargo, la realidad es que en muchas ocasiones no se generan las condiciones equitativas e igualitarias de acceso a los derechos, analizando las necesidades y condiciones de las mujeres. Así, a partir de 1971 el marco del derecho internacional de los derechos humanos de las mujeres empezó a desarrollarse. En el año 2000, la 
Observación número 28, párrafo cuarto, del Comité de Derechos Humanos «insistió que los estados son los responsables de asegurar el disfrute y derechos de hombres y mujeres en condiciones de igualdad y sin discriminación alguna». Señala además, en el párrafo quinto, que «la desigualdad que padecen las mujeres en el mundo en lo relativo al acceso y al ejercicio de sus derechos está profundamente arraigada en la tradición, la historia y la cultura y de esta manera solicita a los estados estar alertas de que no se esgriman a las costumbres y tradiciones como elementos que den pauta a la presencia de condiciones que impiden la igualdad de hombres y mujeres ante la ley».

Aunque la legislación internacional acerca de los derechos humanos de las mujeres es más extensiva (CEDAw, Belem Do Pará), a nivel nacional también se ha hecho un ejercicio jurídico importante sobre el marco de derechos de las mujeres. La Constitución Política mexicana establece en su artículo 1 «la garantía de los derechos humanos de todas las personas, suscritos en la propia Constitución y en Los Tratados Internacionales, también establece la no discriminación por ningún motivo y la obligatoriedad de los estados de promover, respetar, proteger y garantizar los derechos humanos».

Por su parte, la Ley General de Igualdad para Mujeres y Hombres, también es un instrumento que obliga a generar las mismas condiciones desde una perspectiva de igualdad. La Ley General de Acceso de las Mujeres a una vida libre de violencia, establece un derecho fundamental en la vida de las mujeres: el derecho a la no violencia.

A nivel estatal, también se cuenta con la Ley de Igualdad entre Mujeres y Hombres y la Ley de Acceso de las Mujeres a una Vida Libre de Violencia.

Como se puede observar, las distintas legislaciones enmarcan los Derechos Humanos de las Mujeres. Sin embargo, el problema es la eficacia de esos derechos. ¿Cómo podemos hacer que estos derechos puedan ser utilizados por todas las mujeres? Esta cuestión no es exclusiva de poblaciones marginadas. La discriminación y la violencia atraviesan todas las capas de la sociedad. El reto del trabajo de campo es que estos derechos se traduzcan en realidades y se hagan efectivos. El Estado en sus tres niveles - federal, estatal y municipal - tiene una responsabilidad evidente en ello. 
Desde esta perspectiva y la experiencia de trabajo que se tiene con las mujeres, es que consideramos importante comenzar a visibilizar los Derechos Humanos de las Mujeres. Es así como en Redes de Mujeres sin Violencia se habla sobre los Derechos Humanos de las Mujeres en los distintos contextos que viven, tanto en las colonias como en las comunidades, porque ello permite revisar las necesidades existentes.

Uno de estos derechos es el derecho a una vida libre de violencia, que como se mencionó se encuentra establecido en la legislación nacional, local y en tratados internacionales como la Convención Interamericana para prevenir, sancionar y erradicar la violencia contra las mujeres (Convención de Belem Do Para) y la Convención sobre la eliminación de todas las formas de discriminación contra la Mujer (CEDAw).

\section{Violencia contra las mujeres}

Lorena llegó una mañana al Instituto de las Mujeres en plena crisis de ansiedad. Reportaba agresiones verbales de su marido. Mencionaba que las palabras del marido la «hacían sentir mal», como «si no valiera nada», además de los golpes constantes a los cuales ella ya «estaba acostumbrada» y no la dejaba salir a la calle, la vigilaba todo el tiempo, de tal forma que no podía buscar una opción de trabajo que contribuyera a su autonomía. También era obligada a tener relaciones sexuales, porque era «su esposa». En varias ocasiones que estuvo internada, «él acudía y pedía llevársela...».

En el caso anterior, se pueden observar varios tipos de violencia, desde la psicológica, la económica, la física y la sexual. Pero además la grave afectación a la salud mental de Lorena. Lorena refleja los daños que produce la exposición constante a la violencia. Pero, todavía más grave, la violencia que vive Lorena trasciende el ámbito de lo privado. Las afectaciones a la vida de Lorena también se ven reflejadas en su contacto con el resto de las personas. Esto ocasiona que Lorena entre en una dinámica en donde se torna muy fácil victimizarla y abusar de ella prácticamente en cualquier entorno en donde se encuentre. Así, Lorena, como muchas otras mujeres, se convierte en víctima 
silenciosa de innumerables abusos dentro y fuera de su hogar. ¿Cómo detener este ciclo?

La violencia contra las mujeres ya se encuentra debidamente caracterizada. La Organización Mundial de la Salud (oms) define la violencia como «el uso intencional de la fuerza o el poder físico, de hecho o como amenaza, contra uno mismo, otra persona o un grupo o comunidad, que cause o tenga muchas probabilidades de causar lesiones, muerte, daños psicológicos, trastornos del desarrollo o privaciones» (p. 3). Por otra parte, la Organización de las Naciones Unidas (ONU) define la violencia contra las mujeres como «todo acto de violencia basado en la pertenencia al sexo femenino que tenga o pueda tener como resultado un daño o sufrimiento físico, sexual o psicológico para la mujer, así como las amenazas de tales actos, la coacción o la privación arbitraria de la libertad, tanto si se producen en la vida pública como en la vida privada» (Resolución 48/104, 1993).

La CEDAW va más allá de las definiciones y señala principios que pueden tener una mejor operacionalización para el diseño y ejecución de políticas públicas. Al efecto, plantea que la desigualdad y la discriminación de género son perpetuadas por las normas sociales y las desigualdades de poder que se transmiten mediante roles y estereotipos de género establecidos; son causas de la violencia contra las mujeres (violencia de género) y sus efectos deterioran o anulan el desarrollo de las libertades y ponen en peligro los derechos fundamentales de las mujeres así como su integridad física.

Esta visión más operativa permite colocar el derecho de las mujeres a una vida libre de violencia como el centro de acción de las políticas a favor de las mujeres. Se trata de que la política pública incida sobre las estructuras de la sociedad. En palabras de Cubillos (2015), «la interseccionalidad estructural alude a la imbricación de sistemas de discriminación (género, raza y clase social) que tienen implicación específica en las vida de las personas y los grupos sociales» (p. 122). En ese sentido, es importante que la política pública con perspectiva de género, cuestione los sistemas establecidos que han generado la discriminación y desigualdad de las mujeres.

Las líneas de acción del IMMujeres se concentran precisamente en garantizar el derecho de las mujeres a una vida libre de violencia. Un primer paso, obliga necesariamente a documentar el fenómeno. De acuerdo con datos de 
las atenciones diarias del IMMujeres, los tipos de violencia que mayoritariamente afectan a las mujeres podemos observarlos en la tabla 1. Este cuadro nos da un indicador de que la principal violencia que afecta a las mujeres en el municipio, de manera directa, es la psicológica.

Pero como se ha mencionado anteriormente, puede existir la posibilidad que aunque en unos tipos de violencia la estadística es baja, ésta también pueda darse en un conjunto de violencias que afectan la vida de las mujeres. Después de todo, la muestra incluye únicamente las atenciones proporcionadas por el Instituto. Otras fuentes de información municipales, estatales y nacionales nos ayudan a entender mejor la magnitud del fenómeno.

De acuerdo con información del monitoreo diario de medios de comunicación del Instituto Municipal de las Mujeres de León (IMMujeres), en el año 2016, las cuatro principales formas de dar muerte a las mujeres fueron: arma blanca, arma de fuego, golpes y estrangulamiento (IMMujeres, 2016).

En México, según los resultados de la Encuesta Nacional sobre la Dinámica de las Relaciones en los Hogares (ENDIREH) 2016, 30,7 millones de mujeres $(66,1 \%)$ de 15 años y más han enfrentado al menos un incidente de violencia alguna vez en su vida. Además, la encuesta arroja datos sobre los principales ámbitos en los cuales viven esta violencia los cuales podemos apreciar en la tabla 2. Estos datos, nos señalan tres principales ámbitos en los cuales las mujeres viven algún tipo de violencia: el familiar, el comunitario y el laboral.

El aspecto familiar resulta ser un indicador importante, pues denota que a nivel nacional es el ámbito en el que más se vive la violencia y en el municipio de León de acuerdo a datos estadísticos del IMMujeres de enero a septiembre de 2018 se brindaron 2.548 atenciones jurídicas y psicológicas a las mujeres, el ámbito es coincidente. Por otra parte, el espacio comunitario también llama la atención, pues las mujeres no están exentas de ser víctimas de algún tipo de violencia, en la calle, en el transporte público, en los espacios de tránsito cotidiano; por ejemplo, el acoso sexual en las calles por medio de los piropos, las agresiones verbales y tocamientos que pueden ser el inicio de otro tipo de violencia más grave.

En el ámbito laboral la violencia puede darse en el aspecto sexual y en situaciones de discriminación y exclusión de las mujeres; por ejemplo, 
cuando una mujer está embarazada, pareciera que existe un estigma de incapacidad para realizar labores y entonces se puede observar cómo por el hecho de ejercer un derecho, la consecuencia es la vulneración de otro.

Dentro de los tipos de violencias la económica y patrimonial son otros dos aspectos a considerar. En el caso de la primera, se presenta cuando existe el control del ingreso que tienen las mujeres, se les pide que justifiquen lo que han gastado, se les da una cantidad que limita las necesidades de la familia. Cabe mencionar, que este tipo de violencia es tan grave que en muchas ocasiones es una de las principales violencias que impide que las mujeres salgan del ciclo en el que se encuentran, pues uno de los derechos aparejados que tiene es la autonomía económica de las mujeres. La violencia patrimonial se puede presentar, por ejemplo, cuando se retienen a las mujeres documentos que forman parte de su patrimonio, pero también los bienes, cuando se les roban sus artículos y documentos personales.

La violencia sexual que es definida por la oms como «todo acto sexual, la tentativa de consumar un acto sexual, los comentarios o insinuaciones sexuales no deseados o las acciones para comercializar o utilizar de cualquier otro modo la sexualidad de una persona mediante coacción por otra persona independientemente de la relación de esta con la víctima, en cualquier ámbito, incluidos el hogar y el lugar de trabajo» (2013).

En resumen, las mujeres pueden ser víctimas de diversos tipos de violencia al mismo tiempo, en distintos ámbitos; ello implica que desde la teoría de derechos humanos, la interdependencia debe estar presente siempre en los derechos humanos de las mujeres, pues la consecuencia de que una mujer viva un tipo de violencia será que muy probablemente sea víctima de dos o más en distintos ámbitos

La violencia también ocasiona un daño social en el contexto que se presenta. Es por ello que desde la perspectiva social, hablar de manera colectiva con las mujeres sobre el contexto de la violencia y sus efectos puede contribuir a aminorarla. Las Redes de Mujeres no sólo informan sino que también proporcionan herramientas para enfrentar el fenómeno de la violencia desde sus raíces en todos los ámbitos de la sociedad. 


\section{Las Redes de Mujeres sin Violencia}

Ante la situación de violencia contra las mujeres, existe una obligación legal para los Estados de prevenirla, atenderla, sancionarla y erradicarla, por ello se deben implementar políticas públicas y acciones desde una perspectiva de género. El programa Redes de Mujeres sin Violencia surgió en el año 2011 con el objetivo de promover la participación y organización de las mujeres, a través de la conformación de grupos que fomentaran la solidaridad, la seguridad ciudadana, la recuperación de espacios y la cohesión social, con el objeto de prevenir la violencia.

Este programa fue diseñado como una herramienta de prevención de la violencia de manera colectiva, apostándole a la creación de comunidad, lazos de solidaridad y, después, de sororidad para las mujeres, en el cual se pudieran escuchar unas a otras y hablar sobre las necesidades de todas en sus contextos de vida.

En este sentido, las mujeres también se han apropiado de los espacios públicos, por ejemplo en una colonia del municipio, llamada Piletas, existe un área de donación misma que ha sido utilizada por las mujeres para reunirse en los grupos, tanto para los talleres como para actividades diversas que en colectivo llevan a cabo. Otro ejemplo es un centro social de la colonia Valle de Señora, que fue un espacio de mucho uso, incluso como salón de fiestas para las personas de la colonia. Pero éste poco a poco se fue dejando abandonado. Las mujeres adultas mayores de los grupos de Redes de Mujeres lo retomaron y se apropiaron de éste llevando a cabo talleres y cursos de costura. Estas dos experiencias constituyen ejemplos sobre la forma en la que las redes de mujeres se identifican con los espacios y los ocupan para sus encuentros y para realizar actividades con la ayuda del IMMujeres y otras dependencias municipales. Así, los espacios contribuyen a crear un sentido de identidad. ${ }^{2}$

El reto de las ciudades además de proveer de seguridad y canales para el ejercicio de derechos de las mujeres radica en la reapropiación de espacios

2. Para Masolo (2005) la ciudad no es un espacio neutro de género sino que en ella se expresan las relaciones sociales entre hombres y mujeres mismos que se construyen y transforman en el tiempo otorgando lugares para lo masculino y lugares para lo femenino. 
rompiendo con la asignación de género y propiciando la participación activa de las mujeres en la construcción de ciudadanía. Este tipo de ejercicios modifica las dinámicas sociales y culturales que generan y refuerzan los procesos violentos.

Como menciona Ana Falú (2009), las mujeres requieren de ciudades más equitativas e inclusivas porque el espacio público sigue siendo masculino. Las mujeres necesitan un espacio para ejercer ciudadanía activa donde pueda dar vida a los derechos mediante la participación, puesto que, aunque el espacio de mayor inseguridad para ellas sigue siendo el privado, los temores y la existencia de violencias también en lo público restringen su presencia al sentirlo amenazante.

Un punto clave en el sentido del concepto que brinda Ana Falú (2009) es referirse a las ciudades inclusivas para las mujeres. Es decir, que sean miradas desde las necesidades de las mujeres para que se generen las condiciones necesarias en cuanto a la participación activa y el ejercicio de ciudadanía de éstas, desde el aspecto colectivo que permite el diálogo sobre experiencias comunes. Por ello la creación de Redes de Mujeres también se ha convertido en una herramienta que permite a las mujeres ejercer su derecho a la ciudad. ${ }^{3}$

En el Municipio de León entendemos la red como un modelo de organización social donde las personas, en este caso las mujeres, se integran para compartir experiencias, desarrollar competencias y generar lazos con el fin de abonar el bienestar de las mujeres en su comunidad desde la perspectiva de género.

En la implementación del programa Redes de Mujeres sin Violencia intervienen un grupo de promotoras que de manera inicial acuden a las colonias y comunidades a visitar a las mujeres y explicarles el objetivo del trabajo, el cual implica que puedan reunirse en distintos lugares, por ejemplo, espacios públicos que antes no ocupaban para reconocerse en un entorno sororal y reflexionar la posición y situación en que se encuentran a través de dinámicas

3. Para Lozares (1996) las redes son un conjunto delimitado de actores vinculados unos a otros a través de una relación o conjunto de relaciones sociales. Montero (Citado en Lozares, 1996, p. 21) incluye por su parte la existencia de componentes emocionales $\mathrm{y}$ afectivos considerando que en las redes las personas pueden satisfacer necesidades mediante la obtención de apoyo social incluido el soporte emocional. 
educativas sobre diversos temas relativos a los derechos humanos de las mujeres. Cabe destacar que en todos los casos son ellas quienes determinan el espacio y horarios en los cuales se llevarán a cabo los talleres mediante la metodología de educación popular, que permite platicar desde la experiencia y vivencias cotidianas de todas las mujeres, con formas de pensar distintas, de edades diversas y que el logro ha sido que entre ellas se crea un vínculo inclusivo de escucha y de acompañamiento, sin importar las diferencias que tengan unas con otras.

Por ejemplo, contaremos cómo es que se da el primer día de visita:

Las promotoras investigan quién es la presidenta de colonos o dónde se reúnen mujeres o visitan a las y los directores de las escuelas para presentarse como personal del Instituto de las Mujeres. A la persona contactada le hablan acerca de lo que hace el Instituto y del programa de Redes de Mujeres explicando que es un espacio para que las mujeres compartan experiencias, participen en actividades y para que aprendamos acerca de la violencia y los derechos humanos de las mujeres. De ahí se pacta una reunión inicial para que se pueda convocar a otras mujeres - a las mamás de las escuelas o a las vecinas - En la primera reunión que se puede llevar a cabo en algún espacio público, en una escuela, en la casa de alguna de las mujeres o hasta debajo de un árbol, nos presentamos con todas, les preguntamos si conocen que existe un Instituto para las Mujeres y qué han escuchado de él. Generalmente, más que hablar, escuchamos lo que ellas piensan: por qué creen que hay un Instituto para las Mujeres, qué piensan que hace y por qué es necesario un Instituto para las Mujeres. Ahí ellas generalmente expresan que hay machismo, que es necesario porque las mujeres necesitan apoyo o porque las mujeres viven violencia. Entonces nosotras seguimos generando diálogo y preguntas en relación a por qué creemos que hay violencia para las mujeres y machismo y ellas continúan comentando su apreciación. Así nos introducimos, entonces informamos sobre los servicios del Instituto, su creación y los temas que se incluyen en Redes de Mujeres y les preguntamos si están interesadas en participar. Luego pactamos el día y la hora en que nos veremos y les sugerimos invitar a más mujeres. Ya en la primera sesión iniciamos con alguna dinámica para que todas nos presentemos, con las expectativas que ellas tienen del taller y les pedimos que ellas establezcan condiciones necesarias para llevarlo 
a cabo. Así ellas mismas generan las reglas como: confidencialidad, respeto entre otras. Y entonces ya abordamos los temas de cada sesión con sus respectivas actividades vivenciales. Finalizamos la sesión de manera vivencial y plenaria, recordando momentos de su vida en que han sido apoyadas o acompañadas y se comparte con el grupo.

Las sesiones con las mujeres se clasifican en básicas con temas como: el género en todas partes, autonomía de las mujeres, violencia y prevención de riesgos. En las sesiones intermedias nos ocupamos de los temas de derechos humanos de las mujeres, herramientas jurídicas, órdenes de protección, sororidad y organización social.

En las sesiones realizamos dinámicas en las cuales platicamos sobre historias de vida de otras mujeres, historias de violencia, de cómo las mujeres han logrado salir de ahí, de cómo las mujeres participan en la sociedad. Poner estos ejemplos desde lo cotidiano, permite que las mujeres, no se sepan ni sientan solas. Pero también les permite saber que hay salidas viables.

También pretendemos contribuir al empoderamiento de las mujeres, desde los diversos temas que se imparten, la información y las herramientas que se les brinda. Las acompañamos en los distintos procesos y en el caso de que alguna mujer tenga dudas de algún tema específico, hacemos el vínculo con las instituciones, organizaciones o dependencias para que acudan con los grupos de mujeres. Es decir, éstas son otras necesidades que las mujeres nos permiten ver, a través del trabajo de las Redes de Mujeres; por ello es importante destacarlas, porque el contacto que generamos con las mujeres permite construir la confianza entre todas y de esta forma poder colaborar en gestionar acciones en temas relacionados con la salud de las mujeres, la seguridad, la vivienda, entre otros.

Finalmente, hacemos la invitación a las mujeres para que multipliquen la información, algunas de ellas adquieren el papel de «multiplicadoras» siendo enlace directo con el IMMujeres, formando nuevos grupos, transmitiendo información a sus vecinas o incluso, en algunos casos, acompañando a las instituciones especializadas a quienes se encuentran en una situación de violencia. 


\subsection{Logros}

Del año 2011 al 2014 el programa fue financiado con recurso del Subsidio para la Seguridad de los Municipios (SUBSEMUN) de la Secretaría de Seguridad Pública Federal. Los logros durante ese periodo fueron:

Redes de Mujeres para la prevención social de la violencia, la construcción de la paz y la seguridad. Integración de 2 mil mujeres en dos zonas de la ciudad de León (agosto-noviembre 2011).

Se establece una Red institucional para vincularse en la atención a las mujeres víctimas de violencia y se crea un directorio de la equidad con datos de las instituciones municipales dirigidas a la prevención y atención de la violencia. Se adhieren a la Red 4 mil 41 mujeres (diciembre 2011).

Se crea un Plan de acción de la Red de Mujeres con la participación de las integrantes (2012).

Bajo el nombre de «Participación ciudadana de las mujeres para la construcción de una vida sin violencia en el marco de los Derechos Humanos» se crea una campaña de prevención y sensibilización en medios de comunicación, se capacita a funcionarias y funcionarios públicos y se otorgan apoyos para proyectos productivos a mujeres. Durante este periodo se adhieren 3 mil mujeres (2013).

Con el «Programa de apoyo preventivo a mujeres: creación y fortalecimiento de redes» además de la campaña preventiva se integran al equipo un grupo de psicólogas «monitoras» (2014).

En el año 2015, se comienza a trabajar el programa de Redes de Mujeres de manera constante en comunidades rurales de los polos de desarrollo del municipio. Abordando los derechos humanos de las mujeres rurales contenidos en la CEDAw. Se refuerza el Programa Brindando Herramientas Jurídicas a las Mujeres y Acompañamiento en Situación de Violencia.

A partir del año 2014 bajo el programa Redes de Mujeres Indígenas sin Violencia se comenzó a brindar acompañamiento a mujeres indígenas migrantes procedentes del estado de Guerrero que arriban cada año a los campos agrícolas para trabajar en la pizca de chile y tomate. El objetivo principal ha sido 
dar acompañamiento en materia de salud sexual y reproductiva así como ante situaciones de violencia.

En este sentido, Redes de Mujeres ha permitido constatar que la capacitación en perspectiva de género es una herramienta que permite nuevas relaciones entre mujeres y hombres, sobre todo en el uso y ejercicio del poder así como el que nos cuestionemos los modelos masculino y femenino hegemónicos.

Las integrantes de las redes conforme visibilizan situaciones de discriminación o violencia en que ellas o sus compañeras se encuentran deciden, con el acompañamiento del IMMujeres, acercarse a espacios de atención psicológica y legal, a programas de capacitación tanto educativos como para el desempeño de un oficio, programas de emprendimiento o espacios de participación con otras instituciones incidiendo así tanto en su autonomía como en el ejercicio de sus derechos.

Desde el año 2011 hasta el 2017 se han conformado mil 904 grupos, sumado un total de 25 mil 894 mujeres en zonas urbanas. Mientras que a partir del año 2014 se han sumado 172 grupos, con 3 mil 208 mujeres en comunidades rurales y con agrupaciones.

A lo largo de estos años las mujeres que han sido parte de las redes se han acercado a otros programas y apoyos que brinda el gobierno municipal recibiendo cursos de capacitación, apoyos para sus negocios e inclusive han realizado la publicación de un libro en conjunto con el Instituto Cultural de León plasmando sus recetas tradicionales y su visión de la violencia contra las mujeres a través de historias tanto de ellas como de sus vecinas.

\subsection{Evaluación del programa}

Hemos realizado test iníciales y finales de percepción de estereotipos y prejuicios de género considerando los temas abordados. La muestra estuvo integrada por 190 mujeres.

En el test inicial encontramos que las mujeres consideran que hay que dar la razón a sus parejas aunque no estén de acuerdo para evitar problemas. Así respondieron 122 mujeres. El 33\% considera que las mujeres deben darse a 
respetar para no vivir violencia. Lo que centra la responsabilidad de esta problemática en ellas y les re-victimiza. El 12\% de las mujeres consideraba que los hombres son violentos por naturaleza, uno de los mitos más difundidos que exime de responsabilidad a la persona violentadora.

En el test final encontramos que el $96 \%$ de las mujeres consideró que todas y todos debemos dar nuestra opinión y decidir en conjunto. 172 mujeres consideran que ante la violencia las mujeres podemos conocer, exigir y promover nuestros derechos humanos y tener relaciones basadas en la dignidad y el respeto. Además, 182 de las encuestadas piensa que la violencia es un acto con la intención de causar daño y que los agresores saben lo que hacen.

También recogemos algunos comentarios brindados por las mujeres en las encuestas al finalizar las sesiones, entre ellos se incluyen los siguientes:

Para mí fue muy importante saber que tengo los mismos derechos que los hombres, no debo de ser discriminada y que puedo denunciar los maltratos a los Derechos Humanos.

El taller ha llevado mi vida a apreciarme como mujer, a exigir mis derechos y a tomar mis propias decisiones, pero sobre todo a decir no cuando las cosas no están bien.

Tras siete años desde el inicio del programa es posible observar que las mujeres se identifican como parte de las Redes, que hay entusiasmo por seguir siendo parte y acercándose a programas y servicios del IMMujeres y a la par que nos permiten sumar a más mujeres de sus colonias. Es posible encontrar multiplicadoras empoderadas que se vinculan luego con otras instituciones para afianzar el proceso de organización comunitaria y la autogestión.

Como área de oportunidad encontramos que aún se requiere reforzar la capacitación al personal de las instancias de impartición de justicia y seguridad con quienes las mujeres acuden; por ejemplo, cuando después de un taller, deciden interponer un recurso legal ante una situación de violencia, en ocasiones se encuentran con negativa para la obtención de órdenes de protección o con re-victimización en los procesos.

Por otro lado hay mujeres que todavía se ven con limitantes para sumarse a las redes, entre ellas: la inseguridad en algunas colonias o comunidades, 
los mandatos de género que les otorgan responsabilidad casi exclusiva en el cuidado y las tareas domésticas, la idea de que para formar parte de la red es necesario vivir violencia física, el temor a que si las vecinas o conocidas las ven piensen que ellas viven situaciones de violencia o el que todavía haya quienes abandonan el taller porque al notar cambios de actitud son impedidas por sus parejas para seguir acudiendo.

Una tarea pendiente para los próximos años ante el programa es enfocarnos en el empoderamiento económico de las mujeres mediante trabajo transversal con otras dependencias, así como la generación de un protocolo de acompañamiento a mujeres indígenas migrantes para establecer propuestas interculturales que respeten la cosmovisión indígena.

\subsection{Reconocimiento internacional}

En el año 2018, León, Guanajuato, se convirtió en el primer municipio de América Latina en obtener el Premio Internacional Ciudades Educadoras otorgado por la Asociación Internacional de Ciudades Educadoras por el programa Redes de Mujeres sin Violencia en la categoría Buenas Prácticas de Educación Ciudadana a través de la Participación. Se destaca el papel en el empoderamiento de las mujeres en la promoción de la igualdad y la transformación social.

Un aspecto importante del reconocimiento a la experiencia de Redes de mujeres es que el Programa impulsa la participación ciudadana de las mujeres en las colonias y comunidades, por medio de la educación popular, metodología que abre el camino para revisar el sentir y pensar de las mujeres y en consecuencia se constituye como una política pública que visualiza las necesidades de las mujeres en la ciudad.

En este sentido, las Redes de Mujeres sin Violencia tienen ahora el compromiso de difundir la experiencia para que pueda ser replicada en otros espacios y sobre todo continuar el trabajo con las mujeres que viven en el municipio de León.

Una tarea pendiente para los próximos años ante el programa es enfocarnos en el empoderamiento económico de las mujeres mediante trabajo transversal con otras dependencias, así como la generación de un protocolo de acompañamiento a mujeres con cosmovisión indígena. 


\section{Conclusiones}

La violencia contra las mujeres es un problema que a lo largo de los años ha afectado la vida de las mujeres, en los distintos tipos y ámbitos que se expusieron con anterioridad. Y es que cada caso de violencia tiene sus especificidades como se pudo observar también al inicio del artículo y así pueden existir diversos casos.

Aunque consideramos que se ha hecho un esfuerzo en la legislación para tener la perspectiva de derechos humanos de las mujeres, también creemos que aún falta y es que la necesidad de crear y pensar desde la perspectiva de género es una realidad, pese a que los estados tienen el compromiso de legislar a favor de los derechos de las mujeres y las niñas, se tienen que analizar los contextos actuales y reales.

Como se pudo observar también, además de la legislación, se deben implementar acciones para prevenir, atender, sancionar y erradicar la violencia contra las mujeres; desde ese sentido, consideramos que el programa Redes de Mujeres ha sido una eficaz herramienta para visibilizar los derechos humanos de las mujeres en el municipio de León, para ello ha resultado fundamental pensar con y desde las mujeres para acercarnos a ellas y sus contextos. En este sentido lo principal se basa en generar y ganar la confianza de éstas en los trabajos del municipio y del IMMujeres. Esto es producto de un intenso y comprometido trabajo de campo, de cara hacia las mujeres, lo que ha permitido detectar sus preocupaciones, sus problemáticas y detonado otras acciones, tal es el caso del Programa para prevenir y atender la violencia feminicida en el municipio de León.

Por otra parte, la participación de las mujeres dentro del programa ha contribuido a que exijan sus derechos y estén presentes en espacios de las colonias y comunidades en los cuales se discute y habla sobre las necesidades cotidianas, pero también se propone. Las mujeres se han vuelto autogestivas, pues buscan distintas alternativas que les permitan tener mejores condiciones de vida.

Sin duda, consideramos que el programa ha sido una de las prácticas afirmativas municipales focalizada en los derechos humanos de las mujeres, con 
un trabajo de campo que ha permitido conocer las necesidades reales de las mujeres.

\section{Tablas}

Tabla 1. Principales tipos de violencia en mujeres atendidas, en el año 2018, en el IMMujeres.

\begin{tabular}{|l|l|l|l|l|l|l|l|}
\hline Periodo & Psicológica & Física & Económica & Sexual & Patrimonial & Laboral & Bullying \\
\hline Julio-Septiembre & $91 \%$ & $52 \%$ & $32 \%$ & $11 \%$ & $2 \%$ & $0 \%$ & $0 \%$ \\
\hline Abril-Junio & $69.05 \%$ & $33.98 \%$ & $28.57 \%$ & $6.93 \%$ & $4.98 \%$ & $0 \%$ & $.22 \%$ \\
\hline Enero-Marzo & $88.8 \%$ & $38.7 \%$ & $29.4 \%$ & $5.5 \%$ & $2.45 \%$ & $.3 \%$ & $.5 \%$ \\
\hline
\end{tabular}

Fuente: Construcción propia con base en datos del IMMujeres.

Tabla 2. Principales ámbitos en los que las mujeres viven violencia.

\begin{tabular}{|l|l|}
\hline Porcentaje & Ámbito de la violencia \\
\hline $43.9 \%$ & $\begin{array}{l}\text { Han sufrido violencia por parte de su actual o última pareja, esposo o novio a lo largo de su } \\
\text { relación }\end{array}$ \\
\hline $34.3 \%$ & Han experimentado algún tipo de violencia sexual en los espacios comunitarios \\
\hline $26.6 \%$ & $\begin{array}{l}\text { Ha experimentado algún acto violento, principalmente de tipo sexual y discriminación por } \\
\text { razones de género o embarazo en el ámbito laboral }\end{array}$ \\
\hline
\end{tabular}

Fuente: Construcción propia con base en ENDIREH, 2016.

\section{Fotografías}

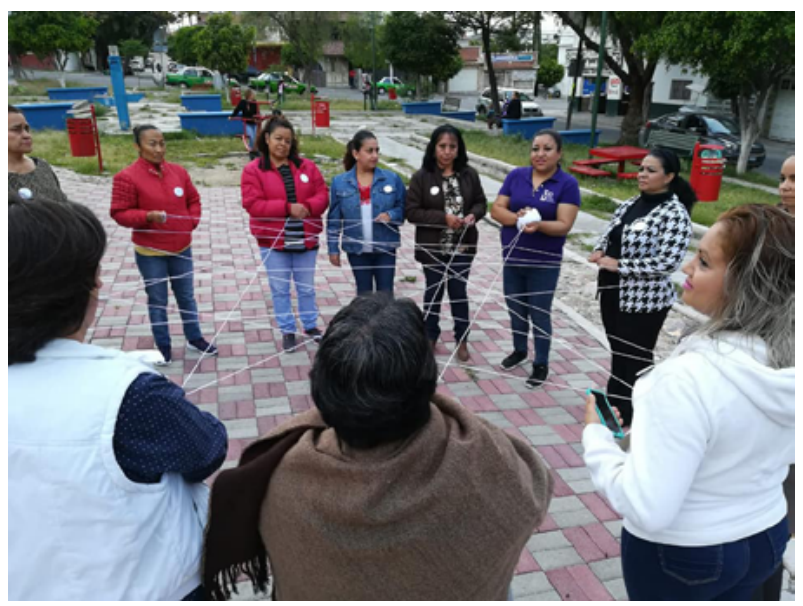

Fotografía 1. Mujeres del polígono de las Joyas en grupo de Redes de mujeres. 


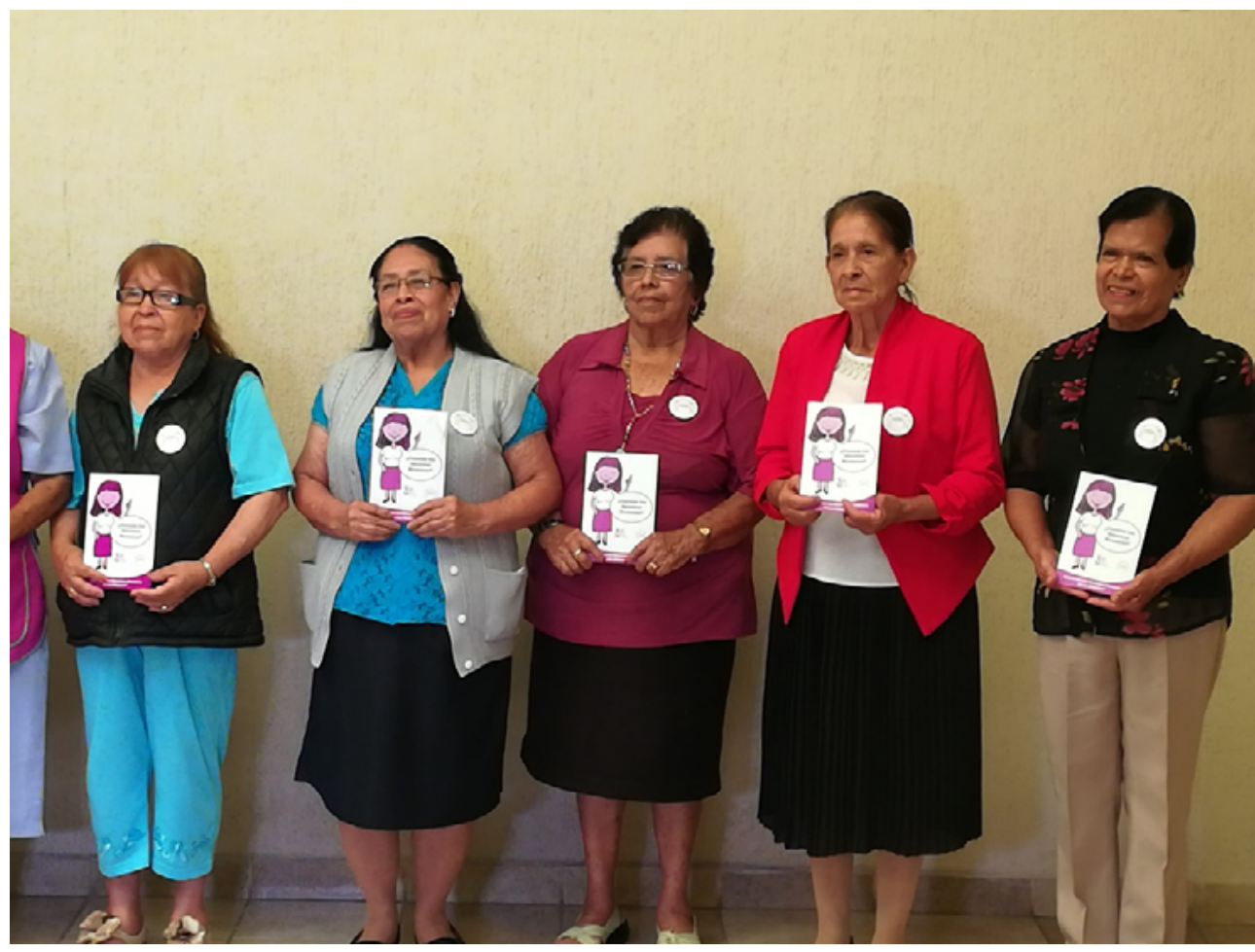

Fotografía 2. Mujeres de Redes de la colonia San José el Alto en cierre de talleres.

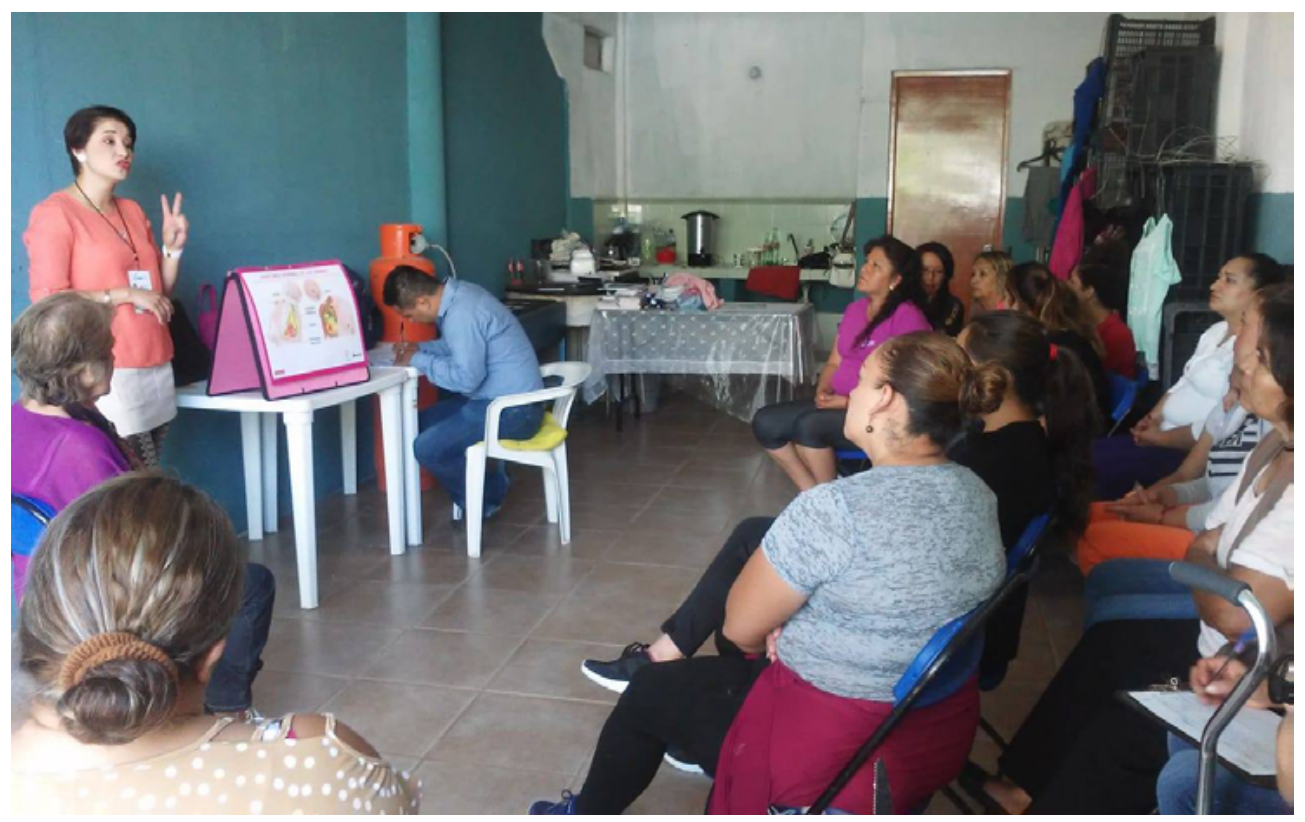

Fotografía 3. Mujeres de Redes en sesión de charla de prevención de cáncer de mama. 


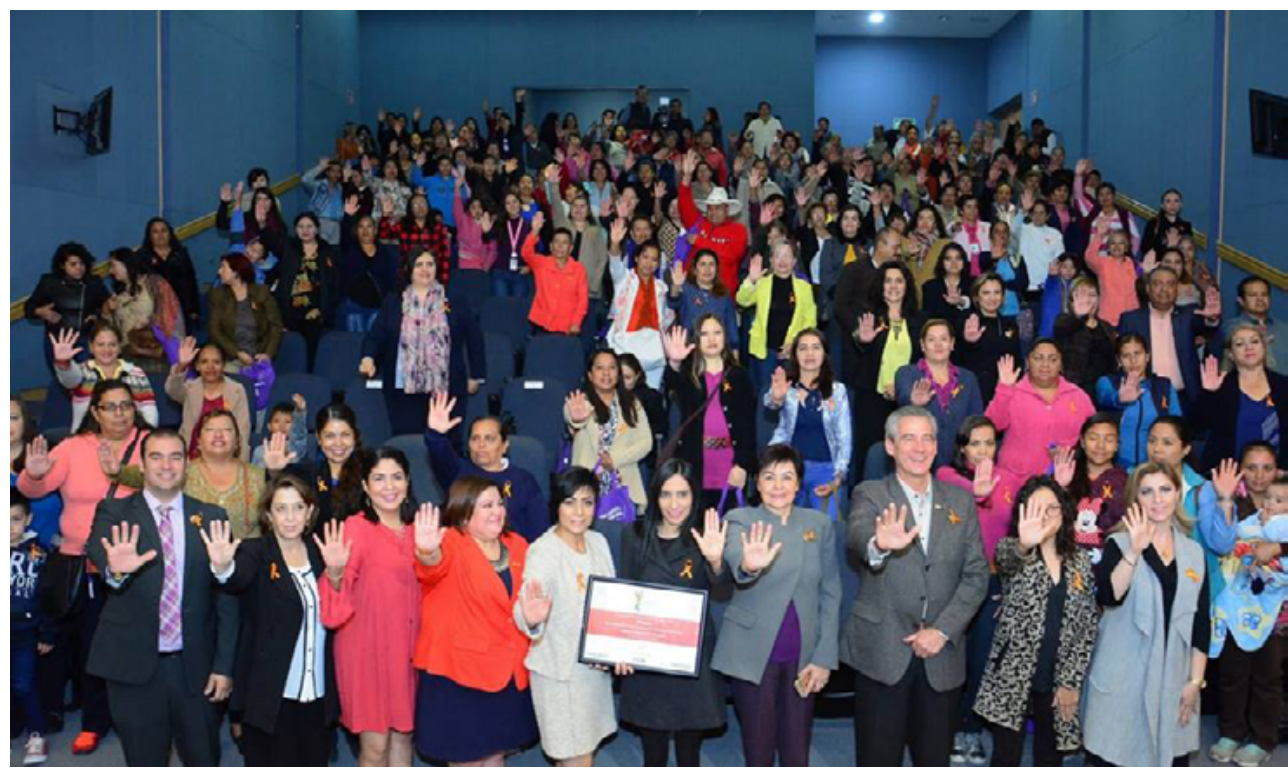

Fotografía 4. Evento conmemorativo por el Día Internacional de la No Violencia Contra las Mujeres y toma de protesta a mujeres multiplicadoras de las Redes de Mujeres sin Violencia de parte del Alcalde de León y la Directora general del IMMujeres.

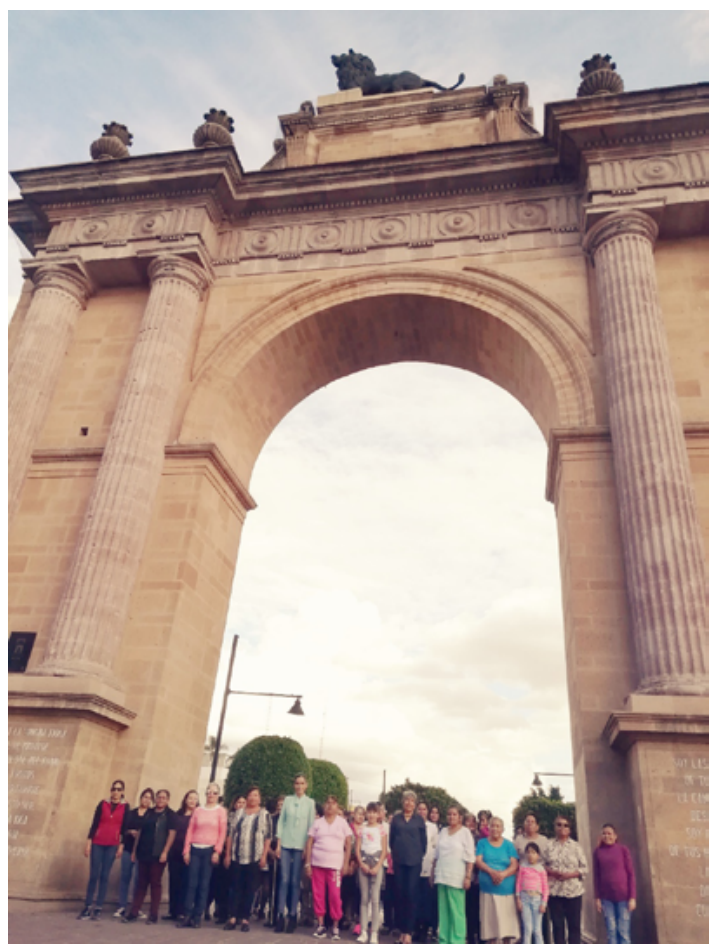

Fotografia 5. Mujeres de las Redes con la Directora, Promotoras y Personal que labora en el IMMujeres, en toma de fotografía para presentación en Cascais, Portugal, para la recepción del premio de la Organización Internacional de Ciudades Educadoras. 


\section{Referencias}

Colombara, M. La violencia urbana explorada desde la perspectiva de género. Experiencias en América Latina. Fundación Propuesta-Argentina. Recuperado de: http://egal2009.easyplanners.info/ area02/2800_Colombara_Monica.pdf

CUBILlos, J. (2015). La importancia de la interseccionalidad para la investigación feminista. Recuperado de: https://www.http://revistes. ub.edu/index.php/oximora/article/viewFile/14502/17834.

FALÚ, A . (2009). Violencias y discriminaciones en las ciudades. En Ana Falú (Ed.) Mujeres en la ciudad. De violencias y derechos. (pp. 15-37) Santiago de Chile, Chile: Ediciones sur.

Instituto Municipal de las Mujeres de León (2016). Diagnóstico situacional de la violencia feminicida en el municipio de León.

Instituto nacional de Estadística y geografía (2016). Encuesta Nacional sobre la Dinámica de las Relaciones en los Hogares (Endireh).

Ley General de Acceso de las Mujeres a una Vida Libre de Violencia para el Estado de Guanajuato. Diario Oficial del Gobierno del Estado de Guanajuato, 26 de noviembre de 2010. Recuperado de:https://portalsocial.guanajuato.gob.mx/sites/default/files/documentos/2013_GOBIERNO_GTO_Ley_acceso_mujeres_vida libre violencia.pdf

Lozares, C. (1996). «La teoría de redes sociales». Universidad Autónoma de Barcelona, Recuperado de: http://ddd.uab.cat/pub/papers/02102862n48/02102862n48p103.pdf

MAssolo, A. (2005): Género y seguridad ciudadana: el papel y reto de los gobiernos locales,Seminario Permanente sobre Violencia, PNUD-El Salvador, Recuperado de:http://es.scribd.com/doc/19779728/Genero-y-Seguridad-Ciudadana-Ponencia-de-Massolo

Organización Mundial de la Salud (2002). Informe Mundial sobre la violencia y la salud. Recuperado de: http://apps.who.int/iris/bitstream/ handle/10665/67411/a77102 spa.pdf;jsessionid=69C2A03FCD125F63C52488AEBA551114? sequence $=1$

Organización Panamericana de la Salud (2013). Comprender y abordar la Violencia contra las Mujeres. Recuperado de: https://oig.cepal.org/ sites/default/files/20184_violenciasexual.pdf 
